\title{
Insulin initiation and intensification in patients with T2DM for the primary care physician
}

This article was published in the following Dove Press journal:

Diabetes, Metabolic Syndrome and Obesity:Targets and Therapy 27 June 2011

Number of times this article has been viewed

\author{
Jeff Unger \\ Catalina Research Institute, \\ Chino, CA, USA
}

Correspondence: Jeff Unger Catalina Research Institute, I4726 Ramona Ave, Suite II0, Chino, CA 91710 , USA

$\mathrm{Tel}+\mathrm{I} 9095908409$

Fax + I 9098995013

Email jungermd@aol.com

\begin{abstract}
Type 2 diabetes mellitus (T2DM) is characterized by both insulin resistance and inadequate insulin secretion. All patients with the disease require treatment to achieve and maintain the target glycosylated hemoglobin (A1C) level of 6.5\%-7\%. Pharmacological management of T2DM typically begins with the introduction of oral medications, and the majority of patients require exogenous insulin therapy at some point in time. Primary care physicians play an essential role in the management of T2DM since they often initiate insulin therapy and intensify regimens over time as needed. Although insulin therapy is prescribed on an individualized basis, treatment usually begins with basal insulin added to a background therapy of oral agents. Prandial insulin injections may be added if glycemic targets are not achieved. Treatments may be intensified over time using patient-friendly titration algorithms. The goal of insulin intensification within the primary care setting is to minimize patients' exposure to chronic hyperglycemia and weight gain, and reduce patients' risk of hypoglycemia, while achieving individualized fasting, postprandial, and A1C targets. Simplified treatment protocols and insulin delivery devices allow physicians to become efficient prescribers of insulin intensification within the primary care arena.
\end{abstract}

Keywords: diabetes, basal, bolus, regimens, insulin analogs, structured glucose testing

\section{Introduction}

Type 2 diabetes mellitus (T2DM) represents a vast and increasing health care crisis in the US. About $90 \%$ of Americans with T2DM are being managed within primary care practices. ${ }^{1}$ Historically, family physicians and internists have preferred to treat patients with oral antidiabetes medications (OADs) rather than insulin. ${ }^{2}$ Currently available insulin analogs may represent an important therapeutic alternative for many patients. Early intensification of diabetes therapies can minimize the risk of long-term complications associated with exposure to chronic hyperglycemia. ${ }^{3}$ The American Diabetes Association (ADA) estimated that in 2007, a total of US\$174 billion was spent managing patients with diabetes, US\$58 billion of which was directed towards managing long-term complications. ${ }^{4}$ Significant costs are attributed to prolonged hospital admissions, frequent outpatient and emergency department visits, and home health care. Diabetes-related illnesses account for $23 \%$ of inpatient costs in the US. ${ }^{4}$

Encouraging primary care physicians (PCPs) to take a more active role in becoming early adopters of insulin therapy would allow more patients with T2DM to experience less glycemic variability and symptomatic chronic hyperglycemia. One question that PCPs should pose to their patients with both prediabetes and diabetes is: what is the primary complication of well controlled diabetes? The answer, of course, is: nothing. Our role as physicians should be to treat patients as quickly as possible, as safely as 
possible, to the lowest glycemic targets as possible, for as long as possible, and to use pharmacologic interventions as rationally as possible.

\section{Overview: physiologic insulin replacement therapy}

The balance between glucose production and utilization is regulated by an integrated network of hormones, neural pathways, and metabolic signals. In the fasting state, insulin secretion is suppressed, leading to gluconeogenesis (glucose production) in the liver and kidneys accompanied by increased glucose generation by the breakdown of liver glycogen (glycogenolysis). In the postabsorptive state, insulin is produced and secreted from the pancreatic $\beta$ cells, inhibiting glycogenolysis and gluconeogenesis while enhancing peripheral glucose uptake and utilization. Insulin also inhibits lipolysis and proteolysis. The net result during euglycemia is that excess glucose is converted into glycogen, triglycerides (TGs), and proteins. When more glucose is present in hepatocytes than can be metabolized or stored as glycogen, insulin converts excess glucose into free fatty acids (FFAs). The FFAs are packaged as TGs in very-low-density lipoprotein, and following plasma transport into adipose tissue, are stored in fat cells to be used as an energy source. ${ }^{5}$

Basal insulin is produced at the rate of approximately 1 unit $(\mathrm{U})$ per hour in order to minimize the effect of hepatic glucose production. ${ }^{6}$ Basal insulin secretion limits lipolysis and FFA production; this may contribute to insulin resistance in the postabsorptive state. The stimulus of eating prompts a 5-10-fold rise in hepatic portal vein insulin concentration that acts to minimize postprandial hyperglycemia. Glucosedependent insulin secretion occurs in two phases. The firstphase insulin response occurs quickly, over a 3-5-minute period $^{5}$ immediately after eating, and ends rapidly, after approximately $5-10$ minutes. First-phase insulin response is genetically predetermined and frequently abnormal in subjects with a first-degree relative with diabetes. ${ }^{7}$ Fifteen minutes after carbohydrates are consumed and the process of digestion begins, a second-phase insulin response is initiated during which $\beta$ cells produce and secrete insulin until all carbohydrates have been absorbed from the gastrointestinal tract and the plasma glucose levels have normalized. ${ }^{5}$ The second-phase insulin response plateaus in about 2-3 hours, yet the postabsorptive states may last up to 6 hours depending on the content of the meal. ${ }^{6}$ Prolonged exposure to even modestly elevated glucose has been associated with $\beta$-cell desensitization, increased apoptosis ( $\beta$-cell death), delays in first-phase $\beta$-cell response to oral glucose, and attenuated second-phase insulin release. ${ }^{8}$

Patients with T2DM have peripheral insulin resistance as well as inadequate insulin secretion by pancreatic $\beta$-cells. During meals, the reduced first-phase insulin response results in postprandial hyperglycemia and a $35 \%$ decrease in hepatic glycogen storage. ${ }^{9}$ A $55 \%$ increase in nocturnal hepatic glyconeogenesis drives excessive glucose production, favoring fasting hyperglycemia and $\beta$-cell decompensation. Based on clinical investigation, observation, and work with animal models, $\beta$-cell decompensation progresses over five stages as shown in Table $1 .^{10}$

Ultimately, genetically prone individuals with progressive $\beta$-cell dysfunction become exposed to an ambient state of chronic hyperglycemia, which becomes unresponsive to the pharmacologic actions of OADs. Successful attainment of the ADA-recommended glycosylated hemoglobin (A1C) target of $6.5 \%-7.0 \%$ can only be achieved with exogenous insulin therapy when $\beta$-cell mass and function has been severely compromised. ${ }^{11}$

The significance of chronic hyperglycemia, even in the prediabetic state, has been demonstrated to cause microvascular and macrovascular complications. In the Diabetes Prevention Program, diabetic retinopathy was observed in $8 \%$ of patients with impaired glucose tolerance (IGT) and increased to $13 \%$ of patients who progressed to diabetes. ${ }^{12}$ Seventy-seven patients with idiopathic peripheral neuropathy demonstrated abnormal glucose metabolism when they received a $75 \mathrm{~g}$ glucose challenge. Fifty-six percent of patients were found to have abnormal oral glucose tolerance testing results, including 26 with IGT and 15 with clinical diabetes. ${ }^{13}$ Other potentially harmful consequences associated with chronic hyperglycemia include oxidative stress, endothelial dysfunction, insulin resistance, hypertension, and intravascular inflammation. ${ }^{14-17}$

\section{Barriers to insulin initiation}

Even modest reductions in $\mathrm{A} 1 \mathrm{C}$ translate into meaningful improvements in economic and medical endpoints. ${ }^{18} \mathrm{~A}$ PCP who does not have access to certified diabetes educators, nurse practitioners, or physician assistants becomes the sole pharmacotherapeutic designer and educator for insulin initiation and intensification. To some busy practitioners, this may present a daunting task. As is frequently the case in clinical practice, insulin initiation is delayed simply because physicians and patients alike are susceptible to misconceptions and fears about disease progression and the role insulin plays. ${ }^{19}$ 
Table I Proposed stages of $\beta$-cell decompensation and dysregulation ${ }^{10}$

\begin{tabular}{|c|c|c|c|}
\hline Stage & $\begin{array}{l}\text { Ambient blood } \\
\text { glucose value }\end{array}$ & $\begin{array}{l}\text { Physiologic changes } \\
\text { within islet/ } \beta \text {-cell }\end{array}$ & $\begin{array}{l}\text { Histologic changes noted } \\
\text { within islet } / \beta \text {-cell }\end{array}$ \\
\hline Stage I & $85-130 \mathrm{mg} / \mathrm{dL}$ & $\begin{array}{l}\text { - Lowered set-point for glucose-stimulated insulin } \\
\text { secretion due to glucokinase activation }\end{array}$ & $\begin{array}{l}\text { - Normal gene expression profile } \\
\text { - } \beta \text {-cell hypertrophy } \\
\text { - } \beta \text {-cell hyperplasia }\end{array}$ \\
\hline Stage 2 & $89-130 \mathrm{mg} / \mathrm{dL}$ & $\begin{array}{l}\text { - Loss of first-phase insulin response } \\
\text { - Near-normal insulin stores }\end{array}$ & $\begin{array}{l}\text { Decreased expression of glucokinase, glucose } \\
\text { transporter proteins, and transcription factors }\end{array}$ \\
\hline Stage 3 & I30-285 mg/dL & $\begin{array}{l}\text { - Loss of second-phase insulin secretion } \\
\text { - Increased ratio of proinsulin: insulin, suggestive } \\
\text { of } \beta \text {-cell injury } \\
\text { - Patient is maximally insulin resistant as } 80 \% \\
\text { of the } \beta \text {-cell function is lost }\end{array}$ & $\begin{array}{l}\text { - Reduced insulin stores within } \beta \text {-cell } \\
\text { - Increased expression of genes which predispose } \\
\text { to loss of } \beta \text {-cell mass and function } \\
\text { - In obese patients, up to } 40 \% \text { of the } \beta \text {-cell } \\
\text { mass is lost }\end{array}$ \\
\hline Stage 4 & $285-350 \mathrm{mg} / \mathrm{dL}$ & - $\beta$-cell apoptosis and death & $\begin{array}{l}\text { - Amyloid and lipid deposits form within islets } \\
\text { - Islets become fibrosed }\end{array}$ \\
\hline Stage 5 & $>350 \mathrm{mg} / \mathrm{dL}$ & $\begin{array}{l}\text { - Marked } \beta \text {-cell destruction } \\
\text { - Loss of signaling between the } \alpha \text { - and } \beta \text {-cell can } \\
\text { increase risk of hypoglycemia }\end{array}$ & - Fibrosis \\
\hline
\end{tabular}

Recent randomized controlled clinical trials demonstrate that insulin treatment can be readily initiated and successfully intensified for many patients within the primary care setting. In fact, insulin-naïve patients have been found to be as adept at intensifying their own insulin regimens as are physicians, and PCPs just as proficient as endocrinologists at titrating basal insulin therapies. ${ }^{20-22}$

Patient fears present a substantial barrier to insulin initiation, and are commonly based on the perception that insulin therapy is a sign of failure. ${ }^{23}$ This concern can be addressed by reassuring patients that the need for insulin arises as a natural consequence of loss of $\beta$-cell function and diabetes progression. Patients who receive education about their individual glycemic goals ( $\mathrm{A} 1 \mathrm{C}<7 \%$; fasting plasma glucose [FPG] 70-130 mg/dL; 2-hour postprandial glucose $[\mathrm{PPG}]<180 \mathrm{mg} / \mathrm{dL}$ ) are more likely to accept insulin therapy as a necessary intervention. ${ }^{24}$

Fear of developing hypoglycemia as well as lack of understanding about how to minimize one's risk and effectively manage this potentially life-threatening adverse event must be addressed prior to initiating insulin therapy. The reported incidence rates for hypoglycemia in T2DM range from 0 to 7.3 episodes per patient-year, depending on the treatment, duration of the disease and the cutpoints used to define severe hypoglycemia; however, when matched for duration of insulin therapy, the frequency of severe hypoglycemia in T2DM is similar to that seen in type 1 diabetes (T1DM). ${ }^{25,26}$ Population-based data suggest that the incidence of severe hypoglycemia necessitating emergency medical treatment in insulin-treated T2DM approaches that in T1DM. ${ }^{27}$ Notably, because the prevalence of T2DM is approximately 20 times greater than that of T1DM, and because most T2DM patients will eventually require insulin treatment, these data suggest that most hypoglycemic episodes, including those of severe hypoglycemia, occur in patients with T2DM.

The fear of hypoglycemia may be mitigated with the use of structured or paired glucose testing which allows patients to visualize pattern recognition of glucose values and better predict whether hypoglycemia is imminent. ${ }^{28}$ Table 2 presents important questions about hypoglycemia that clinicians should ask at every patient visit. Answers to these questions will not only alert clinicians to any deficits in their patients' understanding of hypoglycemia (eg, causes, initiating appropriate treatment),

Table 2 Proactive questions related to hypoglycemia

- When did the event(s) occur? (daytime vs overnight)

- Under what circumstances did they occur? (missed meal, following exercise, excess medication)

-What were the symptoms?

-What was the blood glucose reading?

- How did patient treat the hypoglycemia?

- Did the patient require assistance from another person in order to reverse the hypoglycemia?

- Did the hypoglycemic event re-occur later within a 24-hour period?

-What was done? (eg, carbohydrates ingested, follow-up blood glucose monitoring)

- How soon did hypoglycemia resolve? (Blood glucose levels rose to $>70 \mathrm{mg} / \mathrm{dL}$ )

- How fearful is the patient or the family of hypoglycemia?

- Do they test before driving?

- Do patients "stack insulin" (re-bolus rapid insulin analog within 3 hours of a similar injection)?

- At what glycemic level does the patient perceive hypoglycemia? (If $<50 \mathrm{mg} / \mathrm{dL}$, patient may have hypoglycemia-associated autonomic failure). 
but can provide important insights regarding the potential cause(s) and severity of the hypoglycemia. This information, in turn, will allow clinicians to provide counseling to patients (in-office or referral to a diabetes education program) and initiate necessary changes in patient treatment regimens. Regimen changes may include: adjusting glycemic goals (temporarily or for the longer term) if hypoglycemia unawareness is an issue; use of more appropriate medications; and initiating more frequent blood glucose monitoring or use of continuous glucose sensing for earlier detection of hypoglycemia.

Physician barriers to initiation of insulin include concerns about risks to patients and their competence for selfmanagement of their disease. Time restraints exist within busy practices for teaching patients how to use insulin as well as how to effectively titrate their doses. Insulin can be safely and efficiently initiated within the primary care setting by a designated nursing assistant. Pharmaceutical companies will gladly provide certified diabetic educators who will demonstrate injection techniques to office staff employees and explain various insulin protocols which can be passed on to patients. Group office visits offer another cost-effective means by which patients can learn to initiate and titrate insulin. ${ }^{29}$ With appropriate educational efforts, patients with T2DM can successfully manage basal, and when necessary, prandial insulin therapy, under the care and supervision of PCPs (see Table 3).

The necessity of transitioning to insulin therapy does not imply that a patient has not been adherent to their prescribed lifestyle interventions. Some individuals and physicians will attribute their disease progression as a result of eating too much sugar, consuming too much alcohol, or working too hard and not having time to exercise. The question from patients that frequently arises is, "Just give me a few more months and I know I can turn this all around". One response might be, "When diabetes is poorly controlled, the sugar content of the blood is very high. Sugar is sticky and this sugar sticks to everything; to your eyes, your kidneys, your blood vessels, your nerves, your joints, and your blood. If we don't do something to lower the sugar content of the blood, not only are you likely to begin to feel sicker, you are putting your body at risk for long-term complications. Insulin reduces your blood sugar level and the blood becomes less 'sticky'. The insulins we have today are not like the ones we used to use in the old days. They are much easier to use and you can even choose your own dose. Let's get started today".

Because T2DM is a progressive disease characterized by early impairment of $\beta$-cell function and ultimately loss of $\beta$-cell mass, insulin replacement therapy is required for many patients. Most OADs lose effectiveness over time,
Table 3 Strategies for initiating and titrating insulin for treatmentnaïve patients with T2DM

- Suggest that insulin will help patients achieve glycemic targets and minimize the risk of long-term complications

- Allow patients to actively participate in their insulin dose titration

- Always praise patients on insulin at their visits for their efforts at achieving their glycemic targets

- Remember, patients who are using insulin do not have normally functioning pancreases

- They are performing their own insulin dose calculations, perhaps multiple times each day

- Insulin prescribers should do everything possible to help patients become successful users of insulin

- Individualize therapy to meet the needs of each patient

o Determine which treatment algorithm might work best for every patient

- Emphasize the importance of lifestyle intervention

- This should minimize weight gain and reduce PPG excursions

- Consider group office visits to have patients meet with a certified diabetes educator

- Often, 8-20 patients can be seen at group visits; they are timeefficient and reimbursable by third-party payers

- Provide each patient with an individualized, written insulin protocol to which they can refer

- Prescribe insulin pen devices whenever possible

- Dose titration of insulin is much more accurate with pens than with vials and syringes

- Teach patients how to identify and appropriately manage hypoglycemic events

-When initiating basal insulin, use $0.4 \mathrm{U} / \mathrm{kg} / \mathrm{day}$ as the starting dose - Continue metformin if possible

- If patient requires $>60 \mathrm{U}$ of basal insulin per day, and his or her AIC level is $>7 \%$, add a rapid-acting insulin analog at the largest meal of the day

- The dose for rapid-acting insulin is $0.1 \mathrm{U} / \mathrm{kg} / \mathrm{meal}$

- If AIC level is not reduced to target after 3 months of basal plus bolus insulin, add a second injection at the next largest meal of the day

- Repeat the AIC test at 3 months and if still above target, add a third mealtime injection

- Patients on basal-bolus insulin therapy should consider modified paired glucose testing in order to fine-tune their treatment regimens

Abbreviations: AIC, glycosylated hemoglobin; PPG, postprandial glucose; T2DM, type 2 diabetes mellitus.

requiring frequent monitoring of $\mathrm{A} 1 \mathrm{C}$ levels and adjustment of the treatment regimen to achieve or maintain adequate glycemic control. ${ }^{30}$ As $\beta$-cell function declines further, the use of mealtime insulin replacement therapy must be considered to minimize postprandial hyperglycemic excursions which may impact one's A1C.

\section{Keys to initiating insulin therapy for patients with T2DM in primary care}

Before initiating an insulin regimen, consider the individual's eating, sleeping, exercise patterns and motivation 
for diabetes self-management. Availability of different formulations of insulin and delivery systems permit a great deal of flexibility toward tailoring regimens to a patient's specific needs. Optimally, insulin replacement therapy replicates physiologic insulin secretion in the fasting and postprandial states. Healthy, euglycemic individuals produce sufficient insulin to maintain a plasma glucose level between 85 and $140 \mathrm{mg} / \mathrm{dL}^{6}{ }^{6}$ Thanks to exquisite and complex regulatory mechanisms, plasma glucose levels in healthy people remain within a narrow range throughout wide fluctuations in activity level and food intake. Coordination between pancreatic $\beta$-cell insulin secretion, $\alpha$-cell glucagon secretion, and peripheral insulin action at liver, skeletal muscle, and fat maintains euglycemia, the balance associated with the normal physiologic state.

Patient practice guidelines, such as the consensus panel statement of the American Association of Clinical Endocrinologists (AACE), suggest that insulin therapy should be considered in patients with $\mathrm{T} 2 \mathrm{DM}$ and $\mathrm{A} 1 \mathrm{C} \geq 9 \%$ or for symptomatic patients with $\mathrm{A} 1 \mathrm{C} \geq 8.5 \%$. ${ }^{31}$ Abundant evidence supports the early initiation of insulin therapy, as insulin acts to significantly lower plasma glucose levels while minimizing the long-term complications associated with chronic hyperglycemia. ${ }^{12}$

The Treating To Target in Type 2 Diabetes Study (the 4-T Study), provides some insight into when insulin should be initiated in patients and at what time during the course of their disease prandial insulin might be initiated. ${ }^{30}$ Over 700 individuals with T2DM who were on dual OAD therapy were randomized into this study. One group was randomized to biphasic insulin aspart 70/30 twice daily. The second group was randomized to prandial insulin aspart, three times daily. The third cohort was treated with basal insulin detemir once daily at bedtime. The trial used a clinically relevant protocol with clinic visits every 3 months, a schedule similar to that routinely followed in the primary care setting. After 1 year, patients who continued to have unacceptable rates of hyperglycemia (defined as A1C $>10 \%$ after 1 measurement, 2 consecutive $\mathrm{A} 1 \mathrm{C}$ measurements $\geq 8 \%$ at or after 24 weeks of therapy, or an $\mathrm{A} 1 \mathrm{C}>6.5 \%$ at the end of year 1) were eligible for intensification of their insulin regimens. Therapy with a sulfonylurea was replaced with an additional type of rapid-acting or mixed insulin regimen as follows:

- Aspart was added three times daily to the detemir-initiated arm, starting with $10 \%$ of the current total daily basal dose (minimum of 4 units; maximum of 6 units).

- Detemir (10 units) was added at bedtime to the aspartinitiated arm.
- Aspart was added at midday to the aspart mix 70/30initiated arm starting with $10 \%$ of the current total daily dose (minimum of 4 units; maximum of 6 units). (This regimen is not typically prescribed in the US.)

The primary outcome of the first 4-T Study published in $2007^{32}$ was A1C. There were small but significant differences between the three groups. The group with the highest $\mathrm{A} 1 \mathrm{C}$ was those individuals randomized to basal insulin at bedtime, and those with the lowest A1C had received prandial insulin. However, none of the groups achieved the target $\mathrm{A} 1 \mathrm{C}$ of $<6.5 \%$. As one would expect, basal insulin resulted in optimal reduction of FPG, whereas prandial insulin improved postmeal glucose excursions better than basal or mixed insulins. Less weight gain and fewer episodes of hypoglycemia were noted in the basal insulin cohort. Thus, after 1 year, the 4-T conclusions were as follows: (1) regimens using biphasic or prandial insulin reduced $\mathrm{A} 1 \mathrm{C}$ to a greater extent than basal but were associated with a greater risk of hypoglycemia and weight gain; (2) most patients are likely to require more than one type of insulin to achieve target A1C levels over time, as very few individuals were able to maintain their A1C levels $<6.5 \%$.

Because the investigators noted a progression in the disease process for their patients with T2DM and an inability to reduce $\mathrm{A} 1 \mathrm{C}$ levels, patients were randomized to be placed on prandial insulin. After 2 additional years in the 4-T study, the A1C levels were identical between all three groups. What did reach statistical significance was the fact that those individuals initiated on basal insulin monotherapy at the end of 3 years had less grade 2 or grade 3 hypoglycemia (the more severe forms of hypoglycemia). This group also demonstrated the least amount of weight gain during the 3-year study.

The overall aggregate A1C level at the conclusion of the 3-year 4-T study was $6.9 \%$ and did not differ significantly between treatment groups. However, patients commencingtherapy with basal or prandial insulin more often achieved glycemic targets than those initiating therapy with biphasic insulin. The lowest weight gain and lowest rate of hypoglycemia occurred in the detemir + aspart group, with $63 \%$ of patients achieving $\mathrm{A} 1 \mathrm{C} \leq 7 \%$. Finally, the 4 -T Study supports starting insulin therapy with once-daily basal insulin and adding prandial insulin if glycemic goals are not met within 1 year.

Basal insulin was the most effective treatment regimen within the 4-T protocol because the insulin dose was progressively increased towards specific fasting and postmeal targets. Self-monitored blood glucose values of each subject were analyzed by a computer management system at the time of each visit. An insulin dosing regimen was then prescribed to target 
FPG levels of 72-99 mg/dL and 2-hour postprandial levels of 90-126 mg/dL. Investigators and patients were encouraged to vary suggested insulin doses, as clinically appropriate, and to amend the doses between visits. Hypoglycemia was categorized as grade 1 (symptoms only) if a patient had symptoms with a self-measured capillary glucose level of $56 \mathrm{mg} / \mathrm{dL}$ or more, grade 2 (minor) if the patient had symptoms with a self-measured capillary glucose level of less than $56 \mathrm{mg} /$ $\mathrm{dL}$, or grade 3 (major) if third-party assistance was required. Unfortunately, the computer-generated dosing protocol suggestions are not clinically available to practicing physicians and were used solely for those investigators and patients enrolled in the 4-T study. Nevertheless, initial intensification of therapy in patients with poorly controlled T2DM with basal insulin appears to be a prudent choice. Fasting hyperglycemia contributes more than postprandial hyperglycemia to $\mathrm{A} 1 \mathrm{C}$ levels during periods of poor glycemic control. ${ }^{33}$

Other studies have compared the two basal insulin analogs (insulin detemir and insulin glargine) as background for the addition of mealtime prandial insulin. Two trials sought to demonstrate the noninferiority of insulin determir to insulin glargine in a basal-bolus treatment regimen that included insulin aspart as the prandial component. Hollander and colleagues included 319 patients with T2DM who were randomized to 52 weeks of treatment with insulin detemir or insulin glargine. ${ }^{34}$ Basal insulin doses were adjusted to a prespecified algorithm according to FPG levels. Both regimens produced similar marked decreases in mean A1C levels from baseline ( $-1.52 \%$ for insulin detemir and $-1.68 \%$ insulin glargine). There were no significant differences in the development of hypoglycemia or adverse events. However, patients treated with insulin detemir experienced significantly less weight gain as compared with insulin glargine $(2.8 \mathrm{~kg}$ with insulin detemir, $3.8 \mathrm{~kg}$ with insulin glargine; mean difference -1.04 ; 95\% confidence interval, -2.08 to $-0.01 ; P<0.05$ ).

A similar study included 385 T2DM patients randomized to treatment with either insulin detemir or insulin glargine plus prandial insulin aspart, for 26 weeks. ${ }^{35}$ Both basal insulins produced similar significant $(P<0.001)$ decreases in mean A1C levels at 26 weeks vs baseline $(-1.1 \%$ for insulin detemir; $-1.3 \%$ for insulin glargine). The rates of hypoglycemic events were comparable between groups. Again, the patients randomized to receive insulin detemir experienced significantly less weight gain than did those treated with glargine $(1.2 \pm 3.96 \mathrm{~kg}$ with insulin detemir; $2.7 \pm 3.94 \mathrm{~kg}$ with glargine; $P=0.001)$. These trials confirm that a basal-bolus insulin regimen utilizing either basal insulin, together with the rapid-acting insulin aspart given at every meal, provides safe and effective treatment for effective glycemic control in patients with T2DM.

\section{Intensification of insulin therapy}

Basal insulin therapy may help patients achieve glycemic goals for a time, but predictable and progressive pancreatic $\beta$-cell dysfunction necessitates the eventual addition of mealtime insulin to minimize postprandial hyperglycemia. ${ }^{36,37}$ Glucose fluctuations during the postprandial period elicit more oxidative stress than chronic, sustained hyperglycemia, which can lead to endothelial dysfunction, vascular inflammation, atherosclerosis, and microvascular complications. ${ }^{38,39}$ Pharmacologic interventions with insulin analogs reduce oxidative stress and vascular inflammation and improve endothelial function. ${ }^{40}$ Insulin intensification in patients with T2DM is also cost-effective and has been associated with a marked decrease in health care costs, due to diminished use of OADs, and with significantly fewer inpatient hospitalizations. ${ }^{41}$

Rapid acting insulin analogs exhibit a peak onset of pharmacodynamic activity at 60 minutes postinjection. Peak carbohydrate absorption following a meal occurs at approximately 75-90 minutes after eating begins. Therefore, in order to synchronize the peak activity of insulin with the expected rise in PPG, the analog should be injected 15 minutes prior to the meal unless the blood glucose level is $<80 \mathrm{mg} / \mathrm{dL}{ }^{42}$ This delay between the injection and onset of the consumption of the meal is known as the "insulin lag time". Patients who inject just prior to a meal may experience postprandial hyperglycemia although they have calculated their insulin dose appropriately.

If one is uncertain as to which meal should be targeted for intervention, "structured" glucose testing should be performed for 3 days prior to and 2 hours after each meal. The meal with the highest "delta" (the difference in blood glucose values between premeal and 2-hour postprandial levels) becomes the initial point of intercession.

Choosing the most appropriate meal to initiate prandial insulin may be inconsequential. A study investigating the efficacy of injecting the rapid-acting insulin analog glulisine at either breakfast or at the main mealtime in patients with T2DM on a background insulin glargine plus OADs resulted in significant improvement in A1C levels regardless of meal specificity. ${ }^{43}$

If after 3 months the $\mathrm{A} 1 \mathrm{C}$ is not approaching the recommended target, structured glucose testing should be repeated for 3 days. First, evaluate the "delta" for the meal during which the initial intervention was initiated. The average delta should be $\leq 50 \mathrm{mg} / \mathrm{dL}$, which is considered to be "physiologic". ${ }^{44}$ If the delta is significantly greater than 
$50 \mathrm{mg} / \mathrm{dL}$, consider increasing the baseline dose of prandial insulin at that meal until the 2-hour postprandial glucose level is $<50 \mathrm{mg} / \mathrm{dL}$ from baseline. One could also consider adding a second mealtime injection of a rapid-acting insulin analog at either breakfast or lunch. The initial dose of any prandial rapid-acting insulin can be approximated as $0.1 \mathrm{units} / \mathrm{kg} / \mathrm{meal}$. Thus, a $100-\mathrm{kg}$ person would require $10 \mathrm{U}$ of a rapid-acting analog, which would be injected 15 minutes prior to eating. If the 2-hour postprandial glucose is $\leq 50 \mathrm{mg} / \mathrm{dL}$, the correct amount of insulin was given to cover the carbohydrate content of that meal. However, if the 2-hour postprandial glucose level is consistently $>50 \mathrm{mg} / \mathrm{dL}$, the patient can adjust the mealtime dose of insulin by $1 \mathrm{U}$ per day until the targeted delta is achieved. ${ }^{45}$

A single prandial insulin dose may not allow all patients to reach one's targeted A1C level. Therefore, $>50 \%$ of patients require basal-bolus insulin, implying that injections be given prior to each meal. Several strategies have been implemented in order to develop effective treatment intensification schedules. For example, Meneghini and colleagues evaluated the stepwise addition of prandial insulin aspart in a randomized, controlled, parallel group, open-label, 48-week trial (STEPwise ${ }^{\mathrm{TM}}$ ) that included 296 subjects with T2DM who were inadequately controlled on basal insulin and OADs. ${ }^{46}$ The objective of the trial was to compare sequential addition of insulin aspart to (1) the largest meal with titration based on premeal glucose values, identified as "SimpleSTEP" or addition of insulin aspart to (2) the meal with the largest prandial glucose increment with titration based on postmeal values, labeled “ExtraSTEP”. Following an initial 12-week run-in period during which OADs were continued and basal insulin detemir was optimized, patients had treatment with prandial insulin initiated at their largest meal. After another 12 weeks, patients who had not achieved $\mathrm{A} 1 \mathrm{C}<7 \%$ added aspart at the next largest meal, and such additions were made every 12 weeks among patients who still had $\mathrm{A} 1 \mathrm{C} \geq 7 \%$. By the end of the 48 -week trial, $>75 \%$ of the patients were receiving three insulin aspart injections per day. ${ }^{46} \mathrm{~A} 1 \mathrm{C}$ had decreased by $0.5 \%$ in period 1 , by a further $0.5 \%$ in period 2 , and by $0.2 \%$ in period 3 in both arms (Table 4). At the completion of the trial, there were no statistically significant differences in A1C, hypoglycemia, weight, or treatment satisfaction. The investigators concluded that the SimpleSTEP and ExtraSTEP algorithms are equally effective strategies for intensifying therapy by adding insulin aspart to insulin detemir among patients with T2DM (Table 4).

\section{Choosing the optimal insulin intensification protocol}

Although a basal-bolus insulin regimen (once-daily insulin glargineor insulin detemir plus a rapid-acting insulin analog: lispro, aspart, or glulisine) mimics the physiologic action of endogenous insulin secretion, many individuals may be reluctant to initiate multiple daily injections. Thus, prescribers should match the insulin regimen to the patient's needs, concerns, and requests. As insulin is initiated, the glycemic targets for fasting, postprandial and A1C targets must be addressed. Self-dosing titration algorithms for both basal and prandial insulin should be provided to the patient. Stepwise intensification may promote patient acceptance and enhance adherence with insulin therapy. Insulin titration should be performed using pen devices rather than vials and syringes as pens are simple to use, demonstrate accurate dosing, and can provide titrations of 1,2 , or $3 \mathrm{U}$ per meal with a simple twist of a dial. The 32-gauge needles used in pen delivery systems are virtually painless.

Multiple daily injections of insulin may be required for patients with acute glucose toxicity (FPG $>250 \mathrm{mg} / \mathrm{dL}) .{ }^{47}$ Once the glucose levels normalize, a decision can be made to either maintain insulin intensification, place the patient on an incretin mimetic (glucagon-like peptide-1 receptor agonist or dipeptidyl peptidase-4 inhibitor) or reduce the number of injections the patient is receiving while maintaining the use of OADs.

Table 4 SimpleSTEPTM or ExtraSTEP algorithm ${ }^{46}$

\begin{tabular}{|c|c|c|c|c|c|c|}
\hline Efficacy & Period I & Period 2 & Period 3 & Period I & Period 2 & Period 3 \\
\hline \multicolumn{7}{|c|}{ SimpleSTEP ExtraSTEP } \\
\hline $\mathrm{AIC}(\%)$ & 8.2 & 7.7 & 7.6 & 8.5 & 7.9 & 7.7 \\
\hline FPG (mmol/L) & 8.2 & 7.6 & 7.6 & 8.0 & 7.6 & 7.4 \\
\hline \multicolumn{7}{|c|}{ Hypoglycemia (episodes/yr) } \\
\hline Requiring assistance & 0 & 0 & 0.14 & 0.03 & 0 & 0 \\
\hline \multicolumn{7}{|l|}{ No assistance } \\
\hline$P G<3.1 \mathrm{mmol} / \mathrm{L}$ & 3.5 & 6.1 & 8.8 & 3.3 & 5.5 & 9.3 \\
\hline $\begin{array}{l}\text { Weight change at } \\
\text { end of trial }(\mathrm{kg})\end{array}$ & & 2.7 & & & 2.0 & \\
\hline
\end{tabular}

Abbreviations: AIC, glycosylated hemoglobin; FPG, fasting plasma glucose; PG, plasma glucose. 
Patients who are initiated on basal insulin on a background of OADs can use the Canadian Insight Trial protocol. Basal insulin is initiated at $10 \mathrm{U}$, taken at the same time each night, and increased by $1 \mathrm{U}$ daily until the fasting glucose levels are $<99 \mathrm{mg} / \mathrm{dL} .{ }^{48}$ Lowering fasting glucose levels $>100 \mathrm{mg} / \mathrm{dL}$ will result in concurrent improvement in postprandial excursions, reduce glucotoxicity, and improve $\beta$-cell function. Once fasting glucose levels are normalized, further increasing the basal dosage will not improve any persistent rise in postprandial hyperglycemia. Prandial injections of insulin should be prescribed when $\geq 60 \mathrm{U}$ of basal insulin is required to achieve normalization of fasting glucose or when postprandial glucose values exceed $180 \mathrm{mg} / \mathrm{dL}$ when the patient's morning glucose levels are euglycemic. The $\mathrm{A} 1 \mathrm{C}$ should be monitored again after 3 months. If the $\mathrm{A} 1 \mathrm{C}$ level remains above target, another mealtime insulin injection should be added before the second largest meal of the day. If, after 3 additional months, the $\mathrm{A} 1 \mathrm{C}$ level still remains elevated, a third mealtime injection should be advised.

\section{Conclusion}

Insulin therapy provides many benefits to patients with T2DM. With significant improvements in glycemic control, long-term complications of diabetes and their associated costs may be markedly reduced. Insulin analogs facilitate insulin dosing and reduce the risks of hypoglycemia and weight gain. Patients can readily learn treatment algorithms necessary to safely and effectively reach glycemic targets. PCPs can manage these patients, and referral to specialists may be minimized. Patients failing to achieve glycemic goals with basal insulin and OADs may require addition of prandial insulin. Intensification may be accomplished using a basal-bolus approach, by starting with prandial insulin at every meal, or more gradually, initially targeting one meal, with insulin added subsequently at other meals based on individualized glycemic goals. The safety and efficacy of different intensive insulin treatment algorithms, many of which have been clinically tested within the primary care arena, are clear. These findings should provide PCPs the tools they need to become more effective prescribers of treatments directed towards successful management of patients with poorly controlled T2DM.

\section{Acknowledgments}

The author wishes to thank MedVal Scientific Information Services, LLC for providing editorial assistance. Funding to support the preparation of this manuscript was provided by Novo Nordisk, Inc. Dr Unger received no remuneration for the development of this manuscript. This manuscript was prepared according to the International Society for Medical Publication Professionals' "Good Publication Practice for Communicating Company-Sponsored Medical Research: the GPP2 Guidelines".

\section{Disclosure}

The author reports no conflicts of interest in this work.

\section{References}

1. Centers for Disease Control and Prevention. National ambulatory medical care survey: 2004 summary. Available from: http://www.cdc. gov/nchs/data/ad/ad374.pdf. Accessed June 15, 2011.

2. Hayes RP, Fitzgerald JT, Jacober SJ. Primary care physician beliefs about insulin initiation in patients with type 2 diabetes. Int J Clin Pract. 2008;62:860-868

3. Holman RR, Paul SK, Bethel MA, Matthews DR, Neil HA. 10-year follow-up of intensive glucose control in type 2 diabetes. $N$ Engl J Med. 2008;359:1577-1589.

4. American Diabetes Association. Economic costs of diabetes in the US in 2007. Diabetes Care. 2008;31:596-615.

5. Guyton JR, Hall JE. Insulin, glucagon, and diabetes mellitus. In: Guyton JR, Hall JE, editors. Textbook of Medical Physiology. 11th ed. Philadelphia, PA: Elsevier Saunders; 2006:961-977.

6. Unger J. Physiologic insulin replacement therapy. In: Unger J, editor. Diabetes Management in Primary Care. Philadelphia, PA: Wolters Kluwer Lippincott Williams \& Wilkins; 2007:192-264.

7. Kosaka K, Kuzuya T, Hagura R, Yoshinaga H. Insulin response to oral glucose load is consistently decreased in established non-insulindependent diabetes mellitus: the usefulness of decreased early insulin response as a predictor of non-insulin-dependent diabetes mellitus. Diabet Med. 1996;13:S109-S119.

8. Leahy JL, Bonner-Weir S, Weir GC. Beta-cell dysfunction induced by chronic hyperglycemia. Current ideas on mechanism of impaired glucose-induced insulin secretion. Diabetes Care. 1992;15:442-455.

9. Krssak M, Brehm A, Bernroider E, et al. Alterations in postprandial hepatic glycogen metabolism in type 2 diabetes. Diabetes. 2004; 53:3048-3056.

10. Weir GC, Bonner-Weir S. Five stages of evolving $\beta$-cell dysfunction during progression to diabetes. Diabetes. 2004;53(Supp1 3):S16-S21.

11. Nathan DM, Buse JB, Davidson MB, et al. Medical management of hyperglycemia in type 2 diabetes: a consensus algorithm for the initiation and adjustment of therapy. A consensus statement of the American Diabetes Association and the European Association for the Study of Diabetes. Diabetes Care. 2009;32:193-203.

12. Knowler WC, Barrett-Connor E, Fowler SE, et al. Reduction in the incidence of type 2 diabetes with lifestyle intervention or metformin. N Engl J Med. 2002;346:393-403.

13. Sumner CJ, Sheth S, Griffin JW, Cornblath DR, Polydefkis M. The spectrum of neuropathy in diabetes and impaired glucose tolerance. Neurology. 2003;60:108-111

14. Hartge MM, Unger T, Kintscher U. The endothelium and vascular inflammation in diabetes. Diabetes Vasc Dis Res. 2007;4:84-88.

15. Ceriello A. New insights on oxidative stress and diabetic complications may lead to a "causal" antioxidant therapy. Diabetes Care. 2003; 26:1589-1596.

16. Monnier L, Mas E, Ginet C, et al. Activation of oxidative stress by acute glucose fluctuations compared with sustained chronic hyperglycemia in patients with type 2 diabetes. JAMA. 2006;295:1681-1687.

17. Evans JL, Goldfine ID, Maddux BA, Grodsky GM. Oxidative stress and stress-activated signaling pathways: a unifying hypothesis of type 2 diabetes. Endocr Rev. 2002;23:599-622. 
18. Matza LS, Boye KS, Yurgin N, et al. Utilities and disutilities for type 2 diabetes treatment-related attributes. Qual Life Res. 2007; 16:1251-1265.

19. Riddle MC. The underuse of insulin therapy in North America. Diabetes Metab Res Rev. 2002;18(Suppl 3):S42-S49.

20. Meneghini L, Koenen C, Weng W, Selam JL. The usage of a simplified self-titration dosing guideline (303 Algorithm) for insulin detemir in patients with type 2 diabetes-results of the randomized, controlled PREDICTIVE ${ }^{\mathrm{TM}} 303$ study. Diabetes Obes Metab. 2007;9:902-913.

21. Gerstein HC, Yale J-F, Harris SB, Issa M, Stewart JA, Dempsey E. A randomized trial of adding insulin glargine vs avoidance of insulin in people with type 2 diabetes on either no oral glucose-lowering agents or submaximal doses of metformin and/or sulphonylureas. The Canadian INSIGHT (Implementing New Strategies with Insulin Glargine for Hyperglycaemia Treatment) Study. Diabet Med. 2006;23:736-742.

22. Davies M, Lavalle-Gonzalez F, Storms F, Gomis R. Initiation of insulin glargine therapy in type 2 diabetes subjects suboptimally controlled on oral antidiabetic agents: results from the AT.LANTUS trial. Diabetes Obes Metab. 2008;10(Suppl 2):42-49.

23. Peyrot M, Rubin RR, Lauritzen T, et al. Resistance to insulin therapy among patients and providers: results of the cross-national Diabetes Attitudes, Wishes, and Needs (DAWN) study. Diabetes Care. 2005;28:2673-2679.

24. Cobble ME, Peters AL. Clinical practice in type 2 diabetes: after metformin and lifestyle, then what? J Fam Pract. 2009;58:S7-S14.

25. Hepburn DA, MacLeod KM, Pell AC, Scougal IJ, Frier BM. Frequency and symptoms of hypoglycaemia experienced by patients with type 2 diabetes treated with insulin. Diabet Med. 1993;10:231-237.

26. Holstein A, Plaschke A, Egberts EH. Clinical characterisation of severe hypoglycaemia - a prospective population-based study. Exp Clin Endocrinol Diabetes. 2003;111:364-369.

27. Leese GP, Wang J, Broomhall J, et al. Frequency of severe hypoglycemia requiring emergency treatment in type 1 and type 2 diabetes: a population-based study of health service resource use. Diabetes Care. 2003;26:1176-1180.

28. Polonsky WH, Fisher L, Schikman CH, et al. Structured self-monitoring of blood glucose significantly reduces A1C levels in poorly controlled, noninsulin-treated type 2 diabetes: results from the Structured Testing Program study. Diabetes Care. 2011;34:262-267.

29. Masley S, Sokoloff J, Hawes C. Planning group visits for high-risk patients. Fam Prac Manag. 2000;7:33-37.

30. Holman RR, Farmer AJ, Davies MJ, et al. Three-year efficacy of complex insulin regimens in type 2 diabetes. $N$ Engl J Med. 2009;361: 1736-1747.

31. Rodbard HW, Jellinger PS, Davidson JA, et al. Statement by an American Association of Clinical Endocrinologists/American College of Endocrinology consensus panel on type 2 diabetes mellitus: an algorithm for glycemic control. Endocr Pract. 2009;15:540-559.

32. Holman RR, Thorne KI, Farmer AJ, et al. Addition of biphasic, prandial, or basal insulin to oral therapy in type 2 diabetes. $N$ Engl J Med. 2007;357:1716-1730.

33. Monnier L, Lapinski H, Colette C. Contributions of fasting and postprandial plasma glucose increments to the overall diurnal hyperglycemia of type 2 diabetic patients: variations with increasing levels of $\mathrm{HbA}_{1 \mathrm{c}}$. Diabetes Care. 2003;26:881-885.
34. Hollander P, Cooper J, Bregnhoj J, Pedersen CB. A 52-week, multinational, open-label, parallel-group, noninferiority, treat-to-target trial comparing insulin detemir with insulin glargine in a basal-bolus regimen with mealtime insulin aspart in patients with type 2 diabetes. Clin Ther. 2008;30:1976-1987.

35. Raskin P, Gylvin T, Weng W, Chaykin L. Comparison of insulin detemir and insulin glargine using a basal-bolus regimen in a randomized, controlled clinical study in patients with type 2 diabetes. Diabetes Metab Res Rev. 2009;25:542-548.

36. Liebl A. Insulin intensification - the rationale and the target. Int J Clin Pract Suppl. 2009;63:1-5.

37. Karl DM. The use of bolus insulin and advancing insulin therapy in type 2 diabetes. Curr Diab Rep. 2004;4:352-357.

38. Ceriello A. Oxidative stress and diabetes-associated complications. Endocr Pract. 2006;12(Supp1 1):60-62.

39. Unger J. Reducing oxidative stress in patients with type 2 diabetes mellitus: a primary care call to action. Insulin. 2008;3:176-184.

40. Scognamiglio R, Negut C, de Kreutzenberg SV, Tiengo A, Avogaro A. Effects of different insulin regimes on postprandial myocardial perfusion defects in type 2 diabetic patients. Diabetes Care. 2006;29: 95-100.

41. Aagren M, Luo W, Moes E. Healthcare utilization changes in relation to treatment intensification with insulin aspart in patients with type 2 diabetes. Data from a large US managed-care organization. JMed Econ. 2010;13:16-22.

42. LuijfYM, van Bon AC, Hoekstra JB, Devries JH. Premeal injection of rapid-acting insulin reduces postprandial glycemic excursions in type 1 diabetes. Diabetes Care. 2010;33:2152-2155.

43. Lankisch MR, Ferlinz KC, Leahy JL, Scherbaum WA. Introducing a simplified approach to insulin therapy in type 2 diabetes: a comparison of two single-dose regimens of insulin glulisine plus insulin glargine and oral antidiabetic drugs. Diabetes Obes Metab. 2008;10:1178-1185.

44. Basu A, Man CD, Basu R. Effects of type 2 diabetes on insulin secretion, insulin action, glucose effectiveness, and postprandial glucose metabolism. Diabetes Care. 2009;32:866-872.

45. Unger J. Diagnosis and management of type 2 diabetes and prediabetes. Prim Care. 2007;34:731-759.

46. Meneghini L, Hermansen K, Mersebach H, Svendsen A, Kumar S. Treatment intensification by stepwise addition of prandial insulin aspart to once-daily basal insulin detemir in subjects with type 2 diabetes: the STEPwise $^{\text {TM }}$ trial [abstract]. Diabetes. 2010;59(Suppl 1).

47. Mayfield JA, White RD. Insulin therapy for type 2 diabetes: rescue, augmentation, and replacement of beta-cell function. Am Fam Physician. 2004;70:489-500.

48. Harris S, Yale JF, Dempsey E, Gerstein H. Can family physicians help patients initiate basal insulin therapy successfully? randomized trial of patient-titrated insulin glargine compared with standard oral therapy: lessons for family practice from the Canadian INSIGHT trial. Can Fam Physician. 2008;54:550-558.

49. Raccah D, Haak T, Huet D, et al. Stepwise intensification of prandial insulin versus basal-bolus insulin therapy in patients with type 2 diabetes mellitus [abstract]. 47th European Association for the Study of Diabetes Annual Meeting. 2010:Abstract 958.

Diabetes, Metabolic Syndrome and Obesity: Targets and Therapy

\section{Publish your work in this journal}

Diabetes, Metabolic Syndrome and Obesity: Targets and Therapy is an international, peer-reviewed open-access journal committed to the rapid publication of the latest laboratory and clinical findings in the fields of diabetes, metabolic syndrome and obesity research Original research, review, case reports, hypothesis formation, expert
Dovepress

opinion and commentaries are all considered for publication. The manuscript management system is completely online and includes a very quick and fair peer-review system, which is all easy to use. Visit http://www.dovepress.com/testimonials.php to read real quotes from published authors. 